\title{
La céruse et le blanchiment des villes de brique au milieu du XVIIIe siècle
}

\section{Valérie Nègre}

\section{(2) OpenEdition}

1 Journals

Édition électronique

URL : https://journals.openedition.org/tc/217

DOI : $10.4000 /$ tc. 217

ISSN : 1952-420X

Éditeur

Éditions de l'EHESS

\section{Édition imprimée}

Date de publication : 1 mars 2002

ISSN : 0248-6016

\section{Référence électronique}

Valérie Nègre, "La céruse et le blanchiment des villes de brique au milieu du XVIIle siècle », Techniques \& Culture [En ligne], 38 | 2002, mis en ligne le 11 juillet 2006, consulté le 29 septembre 2022. URL:

http://journals.openedition.org/tc/217 ; DOI : https://doi.org/10.4000/tc.217

Ce document a été généré automatiquement le 29 septembre 2022.

Tous droits réservés 


\title{
La céruse et le blanchiment des villes de brique au milieu du XVIIIe siècle
}

\author{
Valérie Nègre
}

1 La céruse est un produit largement en usage dans le bâtiment aux XVIIIe et XIXe siècles. Elle sert à la confection des mastics de rebouchage, des produits de scellement $\mathrm{du}$ fer $^{1}$ et surtout des trois principales peintures employées dans le bâtiment: la peinture à l'eau (dite "à la colle» ou "en détrempe»), la peinture à l'huile et la peinture à la chaux.

2 Son emploi se développe considérablement dans les villes de brique de la région toulousaine. À partir du milieu du XVIIIe siècle, progressivement dans certaines villes et de façon autoritaire dans d'autres, la peinture à l'huile brun-rouge appliquée sur les parements apparents est abandonnée pour le blanc.

3 L'effet est spectaculaire, les villes méridionales à dominante ocre-rouge virent au blanc. Ce changement dans le choix du coloris des parements correspond à un changement d'attitude à l'égard du matériau: la coloration blanche est motivée par la volonté d'imiter la pierre. Au grand regret de Stendhal, qui se plaisait à retrouver dans ces «belles maisons en briques" sa «chère Lombardie » (Stendhal 1979: 70), la céruse contribue à renouveler l'image des villes méridionales, et à travers elle, celle de toute une société. Cette évolution du goût par rapport au traitement des parements va de pair avec l'amélioration des modes de production du matériau et la baisse de son prix de revient. Évolution du goût, évolution technique, deux phénomènes qui se nourrissent l'un l'autre et contribuent à ce changement.

De la pratique des mélanges au produit « tout prêt "

4 Les définitions du produit dans les ouvrages de construction du XIXe siècle sont révélatrices de certaines pratiques. Morisot $(1804: 15)$ définit ainsi la céruse :

«Du blanc de plomb mélangé avec divers argiles, soit terre de pipe que l'on trouve en Hollande, soit le vrai blanc venant d'Espagne, ou enfin avec du blanc de Troyes, quoique ce dernier soit le moins propre au mélange. [...]. Le blanc de plomb, réuni 
avec un de ces blancs, se broie sous une meule montée verticalement. Cette opération finie, on le moule en petits pains de forme pyramidale, pesant environ deux livres. Ces pains nous sont envoyés enveloppés de papier et ficelés. »

5 On le voit, contrairement à l'usage actuel de ces deux mots, la céruse est donc considérée comme un produit distinct $\mathrm{du}$ blanc de plomb. Cette définition est confirmée par l'entrepreneur de peinture Maviez $\left(1838: 17^{2}\right)$ qui note : «Le blanc de céruse du commerce n'est jamais un carbonate de plomb pur, il contient toujours une plus ou moins grande quantité de substance blanche quelconque ».

6 La proportion de craie ajoutée au blanc de plomb est loin d'être négli-geable, Maviez précise qu'elle varie de $1 / 5$ à la moitié. On trouve dans le principal traité de construction publié dans le Midi de la France, Architecture rurale théorique et pratique, des dosages équivalents allant de $1 / 4$ à la moitié ${ }^{3}$.

7 La céruse n'est donc pas un produit unique mais un ensemble de produits à base de mélanges de blancs, que les manuels désignent sous le nom de "blanc de craie", "blanc de Troyes", "blanc de Champagne " et "blanc d'Espagne» (équivalent du «blanc de Bougival» ou de «Meudon »). Morisot cite trois types de peintures blanches à l'huile et six types de peintures blanches «à la colle »; mais dans les faits, la variation des dosages, comme celles des différents blancs, donnent une gamme de produits très étendue. Il en va de même pour la peinture à la chaux, qui constitue la troisième grande catégorie de peinture employée dans le bâtiment. Un devis de construction de 1819, concernant le palais de justice de Villefranche (Haute-Garonne), confirme la présence de céruse : «[...] le plancher du palais de Justice sera reblanchi de trois couches, ces trois couches seront composées moitié d'un lait de chaux et l'autre moitié d'un blanc de sérruze $»^{4}$.

8 Un des facteurs déterminants dans la pratique de ces mélanges est le coût. Jusqu'au début du XIXe siècle, le blanc de plomb pur est beaucoup plus cher que la céruse. En 1804 , broyé à l'huile, il vaut approximativement le double du prix du blanc de plomb coupés. En comparaison, les autres blancs sont beaucoup plus économiques, respectivement trois et quatre fois moins cher pour le blanc de Troyes et le blanc $\mathrm{d}^{\prime}$ Espagne ${ }^{6}$. Le blanc de craie a aussi un effet sur les caractéristiques techniques du produit : il augmente son pouvoir couvrant ${ }^{7}$. On peut penser que le temps de séchage et l'odeur ont aussi leur importance; d'après la même source, et pour la peinture à l'huile, la céruse est le mélange qui sèche le plus vite (en 24 heures). Il faut compter le double pour la peinture au blanc de plomb, quatre jours pour le blanc de craie et cinq pour le blanc de Bougival (Morisot 1823, III : 40).

Aux mélanges opérés par les fabricants s'ajoutent les mélanges réalisés par les entrepreneurs sur le chantier. Unanimement déconseillés par les auteurs de manuels techniques, dans le cas de peintures à l'huile, ils sont pourtant courants. En témoignent les très nombreux commentaires qui leur sont consacrés et la parution du Recueil de notes sur les abus de Leclaire (1841). Déjà en 1786, Le Camus de Mézières condamnait sévèrement ces pratiques :

« On ne doit jamais se servir de gros blanc, ou blanc d'Espagne broyé à l'huile, il fait de très-mauvais ouvrages, il faut le réserver pour les détrempes. Le blanc de céruse est le seul dans le genre. » (1786, II : 42).

Le blanc d'Espagne est pareillement proscrit par Miché qui donne un moyen de reconnaître la présence de molleton, c'est-à-dire de blanc de craie broyé à l'huile (1825 : 383) ${ }^{8}$. Mais le traité de peinture de Maviez donne des précisions sur ces mélanges 
opérés par les entrepreneurs : certains blancs comme les blancs de Champagne et de Troyes peuvent être mélangés à l'huile tandis que d'autres, comme les blancs d'Espagne, de Meudon ou de Bougival, sont à proscrire. Les inconvénients sont amplement décrits: «[...] ces blancs ne couvrent pas et pelotent sous la brosse » (Maviez 1820:12). «Les peintures exécutées avec ces produits deviennent noires, s'effritent et n'ont aucune solidité ». (Guédy 1912: 435) ${ }^{9}$. Les mélanges sont donc possibles mais dans le respect de certaines règles.

11 La pratique des mélanges renvoie ainsi directement au problème du contrôle. Comment vérifier la qualité des blancs ajoutés par les entrepreneurs? Dans son Guide des constructeurs, Mignard (1847, I : 487) souligne :

«Les difficultés d'apprécier justement les travaux de peinture, difficultés beaucoup plus grandes à surmonter que toutes celles qu'offrent les autres parties du bâtiment, où tous les matériaux se montrent, pour ainsi dire, à découvert [...]. Avec des matières bien inférieures, et avec plus ou moins de couches, on peut produire, pour un temps assez court à la vérité, les mêmes tons, le même prestige qu'avec les meilleures. »

12 La distinction entre peinture "à la colle » et peinture à l'huile n'était pas évidente, même pour les architectes et les vérificateurs ${ }^{10}$. En ce qui concerne la composition des blancs, on peut penser que la distinction était encore moins aisée. C'est en soupesant à la main les différents pots que les architectes étaient censés reconnaitre les produits (Janniard $1849: 34)$. On comprend dès lors l'intérêt que les maîtres d'œuvre et les maitres de l'ouvrage avaient à remplacer ces produits composés par des produits simples.

13 C'est vers des produits purs qu'on s'achemine à partir des années 1840 . On note une évolution rapide par rapport à la pratique de couper le blanc de plomb, non pas pour les peintures à la chaux et à l'eau, mais pour celles à base d'huile et notamment pour celles qui sont utilisées en extérieur. Les livres de construction témoignent de cette évolution. La distinction entre blanc de plomb et céruse disparaît vers le milieu du siècle, comme le montre le titre même du paragraphe «Blanc de plomb ou céruse », du manuel Technologie du bâtiment (Château 1866) ${ }^{11}$. Au début du XXe siècle, les consignes sont claires :

«Le propriétaire doit exiger que la céruse arrive au chantier en baril cacheté, avec une marque de fabrique: Théodore Lefranc ou Besançon, mais en tout cas, une marque connue; car, sous le couvert d'appellations générales, on voit employer des marchandises qui n'ont aucune parenté avec la céruse. » (Guédy 1912 : 435).

On apprécie les «marques de fabrique » et non plus la variété des produits et la variété des prix qui en découlait. C'est à la mise en place de produits simples, ancêtres des gammes « prêt à l'emploi » d'aujourd'hui, que l'on assiste alors.

Un produit de maquillage pour le bâtiment

On peut suivre l'évolution de ces pratiques dans le Midi de la France, mais deux facteurs supplémentaires amènent un développement particulier de la céruse: l'usage de peindre les parements de brique à l'huile et l'abandon des teintes brun-rouge ou " ciment de brique » au profit du blanc ${ }^{12}$. Les devis de construction des XVIIe et XVIIIe siècles le montrent clairement: les façades en briques et les parties en pierres apparentes étaient peintes à l'huile dans le cas de constructions de choix, la peinture à la chaux, moins résistante, étant réservée aux constructions modestes ou aux parements enduits. Un devis de 1783 prévoit : 
«L'entier parement vu extérieur tant dudit frontispice que de la tour et flèche du clocher sera peint de trois couches de couleur à la séruse broyée avec de l'huile de lin cuite avec de la litharge d'or, le mini, le brun-rouge, la terre d'ombre et des gousses d'ail, on formera la teinte la plus convenable pour imiter la pierre de taille... $\|^{\prime 3}$

16 Les murs latéraux enduits sont en revanche peints "à fresque » avec une peinture à la chaux. Le portail en pierre de l'église du Taur, restauré en 1768, est peint « [...] au blanc de céruse à trois couches mêlé d'une teinte roussâtre $»^{14}$. De la même manière, un bail à besogne de 1746 mentionne un parement apparent en brique peint «à l'huile de noix ou lin et brun rouge [...] à l'exception toutefois de la corniche et cordon du mur qui seront peints en blanc à trois couches dont la première sera à la cole avec du blanc d'Espagne et les deux autres seront à l'huile et à la céruse, le tout fileté à façon de pierre avec de la couleur grise $»^{15}$. Pour les façades en briques apparentes, et jusqu'au milieu du XVIIIe siècle, on se servait donc de peinture à l'huile de noix ou de lin. Cette pratique était aussi courante en Italie. Verdier \& Cattois (1855, I : 53) notent qu'à Sienne, au milieu du XIXe siècle, la brique est laissée apparente ou recouverte d'une couche d'huile de lin renouvelée à des intervalles réguliers de dix ans.

Ce mode de traitement des parements appelle deux remarques. La première, c'est que le choix de la peinture à l'huile plutôt que du badigeon était lié à la recherche d'une protection plus durable pour les façades. On peut penser que les dix années mentionnées par Verdier \& Cattois corres-pondent à peu près à la durée de vie de cette couche protectrice. Parmi les trois grands types de peintures -à la colle, à la chaux et à l'huile-, c'est sans conteste la peinture à l'huile qui était considérée comme la plus résistante. La deuxième remarque concerne la couleur. L'utilisation du brun-rouge ou $\mathrm{du}$ « ciment rouge $»^{16}$ mentionné dans les devis, témoigne, à n'en pas douter, d'un goût pour la couleur rouge et au-delà, pour la terre cuite en général.

Un changement d'attitude se fait jour pourtant à la fin du XVIIIe siècle. Le goût pour la couleur brique disparaît au profit du blanc, légèrement teinté d'ocre de rut ou de gris de perle. Une description de la ville de Montauban, datée de 1773, rend compte de ce phénomène naissant :

«Montauban est une ville enfumée, bâtie en briques, excellentes à la vérité, mais qui, faute d'être rougies de temps en temps, prennent un air noir. Peu de maisons sont crépies et blanchies, excepté celles qu'on reconstruit à présent ou qui le sont depuis peu... » (cité par Costa 1983).

19 À Toulouse, ce parti est imposé par une délibération municipale du 15 juin 1783, obligeant les propriétaires qui font construire ou réparer leurs habitations à blanchir leurs façades. Comme le texte le laisse entendre, le règlement vient entériner une pratique déjà répandue :

« Déjà divers propriétaires, des architectes et des ouvriers ont reconnu le bien fondé de cette méthode, car si l'on parcours la ville, l'on voit un grand nombre de maisons dont les dehors sont crépis, peints à la céruse, ou au lait de chaux, enduit de stuc ou de badigeon. "

20 Le changement est radical : l'usage du badigeon ou de la peinture blanche s'applique alors à tous les édifices, aussi bien à ceux nouvellement construits qu'à ceux conçus à l'origine en briques apparentes. La place de Montauban est ainsi badigeonnée en blanc pour la venue de Napoléon en 1808, de même que de nombreux hôtels toulousains. Ce phénomène est amplifié à Toulouse par un décret du 26 mars 1852 qui prescrit que les façades des maisons doivent être «tenues en bon état de propreté [...] grattées, 
repeintes, ou badigeonnées, au moins une fois tous les dix ans, sur l'injonction qui sera faite au propriétaire par l'autorité municipale ». Plusieurs raisons officielles justifient ce changement. Le règlement toulousain évoque en premier lieu la sécurité :

«Si l'on veut éviter les crimes de nuit dans les rues de la ville, il faut en tenir les rues éclairées, durant les nuits de l'année où la rigueur des saisons qui concentre les habitants chez eux, les rend désertes et favorise les crimes [...]. La blancheur des murs réfléchit pleinement la lumière du jour, ce qui rend les Rues et les Maisons plus claires, plus agréables, les Habitations plus saines, plus commodes, et fait des Perspectives plus belles que celles qu'offre le ciment rouge qui est en usage. » (cité par de Gorse 1942 : 79).

La peinture blanche est aussi considérée, on le voit, comme un facteur d'embellissement et d'assainissement. Mais on l'apprécie surtout en ce qu'elle offre l'avantage de donner à la ville l'apparence de la pierre. Un deuxième texte (12 juillet 1783) évoque l'éloignement des carrières de pierre : « [elles] réduisent la ville à la seule ressource d'imiter ce genre de construction [en pierre] plus propre à faire ressortir le bon goût et la véritable architecture» (de Gorse 1942: 79). Ce goût nouveau pour la pierre est général. Depuis les fabricants de briques, qui tentent de produire des briques et des produits de terre cuite blancs, couleur "du marbre le plus pur ", jusqu'aux peintures de parement, on peut dire que l'on recherche, en ce début du XIXe siècle, une apparence de pierre. Produit de base du maquillage des acteurs au théâtre, la céruse sert désormais de maquillage aux bâtiments de brique.

« Nouvelles couleurs, nouvelles jouissances »

La portée de ces changements de ton et d'apparence est loin d'être anecdotique. Le Camus de Mézières attire l'attention sur les effets de telles transformations: « nouvelles couleurs, nouvelles jouissances », déclare-t-il (1786). La peinture susciterait de nouvelles manières de vivre. Elle est, à ses yeux, responsable de la "gaité des appartemens, si précieuse à nos sensations, et en même temps plus nécessaire qu'on ne pense pour la santé » (Le Camus de Mézières 1786, II : 38). La peinture procure agrément, et par la même bien-être et santé. C'est en tout cas la perception et l'image de toute la société qui se trouvent affectées par ce changement de couleur. En témoigne l'aversion de Stendhal pour la couleur «café au lait " qui recouvre les façades de brique. Avec cette couleur, c'est la parenté des villes méridionales avec les villes de l'Italie centrale que l'écrivain voit s'estomper : «La brique engage à se départir de la laideur gauloise " (Stendhal 1979: 70). C'est aussi la revendication d'une "nationalité méridionale» qui se joue là au profit d'une identité plus «française» ou « internationale ». Le goût pour la couleur blanche de la pierre n'est-il pas l'expression directe du goût néoclassique, ce «style international » qui met momentanément fin aux particularismes locaux ? ${ }^{18}$

Le règlement toulousain affirme clairement ce changement de ton comme une affaire de goût et de santé publique. La mode est ainsi un facteur déterminant du développement du produit dans la mesure où son usage est nettement accru par l'adoption de tons blancs.

24 Mais d'autres facteurs non imposés par le règlement sont aussi déterminants. L'évolution du prix de la céruse notamment. Dans la Haute-Garonne, le prix de la peinture à l'huile à base de céruse baisse de moitié entre 1800 et 1850 , passant de $1,90 \mathrm{~F}$ en 1821 à $0,90 \mathrm{~F}$ en 1850. Contraints de ravaler leurs façades tous les dix ans (par le règlement de 1852), les propriétaires ont intérêt à utiliser des produits plus résistants que les badigeons. La revue Le propagateur des procédés industriels dans le Midi de la France 
(1828: 143) témoigne bien de ces préoccupations liées à la durée des peintures à la chaux. Elles perdent, note le commentateur "leur fraîcheur » et «leur netteté ». La revue présente un badigeon "propre à conserver aux anciens bâtimens toute leur fraîcheur ", expérimenté à Paris, dans la cour du Louvre et à base de chaux vive, de plâtre cuit et de céruse. L'habitude de peindre les façades à l'huile se développe de manière générale en France au XIXe siècle, malgré l'avis de certains spécialistes comme l'architecte Janniard qui dénonce non sans exagération « l'application in-considérée de ce soi-disant préservatif, qui modifie si malheureusement la physionomie sévère et vigoureuse des édifices de pierre de taille, et leur donne l'aspect mou et blafard d'une masse de beurre " (Janniard 1842: 26). Un autre facteur important, bien que moins apparent, souligné plus haut, est celui du contrôle. Le blanc de plomb se présente au début du XIXe siècle comme un produit résistant, qu'il devient possible d'utiliser pur grâce à la baisse de son prix de fabrication- et, partant, dont on peut plus aisément contrôler la qualité.

Les caractéristiques techniques de la céruse et les progrès introduits dans les modes de production ont ainsi favorisé le développement de la pratique du blanchiment, même si l'évolution du goût semble être l'élément le plus déterminant. Ces facteurs expliquent pourquoi, dans une France où les fabriques de céruse sont concentrées au Nord, Toulouse fait exception avec ses trois fabriques locales.

\section{BIBLIOGRAPHIE}

Borromée

1867 « Études sur l'exposition universelle de 1867. Peinture », Gazette des architectes du bâtiment :

109-118.

Chateau, Théodore

1866 Technologie du bâtiment ou étude complète des matériaux de toute espèce employés dans l'art de bâtir. Paris : A. Morel.

Coppolani, Jean

1983-84 « Une œuvre de l'architecte toulousain Philippe Hardy, l'église de Seysses-Tolosane », Mémoires de la société archéologique du Midi de la France XLV : 160-176.

Costa, Georges

1983 « Briques apparentes et enduits dans l'architecture traditionnelle de la région toulousaine », Revue de l'Art 58-59 : 115-122.

Daly, César

1843 « Dialogues sur la concurrence sans limite dans la peinture de bâtiment », Revue générale de l'architecture et des travaux public , t. IV, col. 379-384.

Gorse, Pierre (de)

1942 «Comment nos façades roses devinrent-elles blanches? », l’Auta 135 : 79-81. 
Guédy, Henry

1912 La Construction moderne pratique. Paris : Garnier frères (2ème édition).

Janniard, Henri

1842 « Sur le moyen d'empêcher l'humidité du sol de monter dans les murs des maisons », Revue générale de l'architecture et des travaux publics, t. III, col. 23-26.

1849 « Peinture au blanc de zinc », Revue générale de l'architecture et des travaux publics, t. VIII, col. 31-35.

Le Camus de Mézières, Nicolas

1786 Le Guide de ceux qui veulent bâtir. Paris : L'auteur.

Leclaire, Edmé-Jean

1841 Recueil de notes sur les abus introduits dans la peinture en bâtiment, ainsi que dans la dorure, la tenture et la vitrerie. Paris : Carilian-Gœury et Dalmont.

Maviez, R.

1838 Traité complet, théorique et pratique de la peinture en bâtimens, de la vitrerie, de la dorure, de la tenture de papiers. Paris : L'Auteur (2ème édition).

Miché, Alexandre

1825 Nouvelle architecture pratique ou Bullet rectifié et entièrement refondu. Paris : l'Auteur (1ère édition 1812).

Mignard, B.-Raillard

1847 Guide des constructeurs ou traité complet des connaissances théoriques et pratiques relatives aux constructions. Paris : l'Auteur.

Morisot, Joseph

1820-24 Tableaux détaillés des prix de tous les ouvrages de bâtiment divisés suivant les différentes espèces de travaux. Paris : l'Auteur (1ère édition 1804).

Stendhal

1979 Dans le Midi, de Bordeaux à Marseille. Paris : Éditions Encre (ouvrage publié à partir du manuscrit du Voyage dans le Midi [1838] resté inachevé).

Saint-Félix de Mauremont, Armand (marquis de)

1858 Architecture rurale, théorique et pratique, à l'usage des propriétaires et des ouvriers de la campagne. Toulouse : Douladoure (1ère édition 1820).

Verdier, Aymar \& François Cattois

1855-57 Architecture civile et domestique au Moyen Âge et à la Renaissance. Paris : Didron.

Vitry, Urbain

1828-30 Le Propagateur des procédés industriels dans le Midi de la France. Toulouse : Imprimerie de Benichet Cadet.

\section{NOTES}

1. Les devis de construction donnent parfois la composition de ces produits. Un devis de 1841 précise ainsi : « Les scellements du fer seront faits avec un mélange de céruse, de limaille de fer et soufre par parties égales réduite en pâte consistante au moyen d'un peu de lait écrémé, le tout fondu à une chaleur douce et employé chaud » (Devis pour la construction d'une prison à Gaillac, 1841, Archives Départementales du Tarn 4N 91). Pour le mastic, l'ouvrage de l'architecte toulousain Urbain Vitry (1828-30 : 223-224) 
donne la composition suivante «[...] blanc d'Espagne réduit en poudre avec une certaine quantité de céruse, incorporés sur le marbre avec l'huile de lin et mis en consistance de pâte ».

2. Nous n'avons trouvé aucun exemplaire de la première édition de cet ouvrage.

3. «Les blancs qu'on emploie pour la peinture, sont un mélange de céruse et de blanc d'Espagne, dans les proportions suivantes : pour la peinture en détrempe, on se sert du blanc d'Espagne, seul ou mêlé de 4 onces par livre, et jusqu'à une quantité égale de blanc de céruse. Pour la peinture à l'huile, on se sert de la céruse seule, ou on la mêle avec du blanc d'Espagne, dans la proportion d'un quart à la moitié. » (Saint-Félix de Mauremont $1858: 161$ ).

4. Devis pour des réparations à faire au palais de Justice de Villefranche, 1819. Archives Départementales de la Haute-Garonne, 4N 135.

5. Soit $1,70 \mathrm{~F}$ la livre de blanc de plomb pour $0,92 \mathrm{~F}$ la livre de blanc de céruse. Les prix donnés correspondent aux produits broyés à l'huile. Le broyage augmente considérablement le prix. Il faut quatre heures pour broyer une livre de blanc de plomb en écaille avec de l'huile, une heure quarante pour broyer du blanc de craie ou blanc de Troyes, et seulement $50 \mathrm{mn}$ pour du blanc d'Espagne, de Bougival ou de Meudon (Morisot 1823, III : 69). Dans le prix de revient au mètre carré de peinture, entre également un autre facteur : le foisonnement de la matière colorante. Il faut par exemple trois fois moins de blanc de céruse que de blanc de craie pour couvrir une toise carrée de peinture.

6. Broyé à l'eau, la différence est encore plus importante : le blanc de plomb pur est trente fois plus cher que le blanc d'Espagne. Toujours d'après Morisot, le blanc de craie ne coûte que 0,58 F et le blanc d'Espagne ou de Bougival, 0,36 F la livre. Broyé à l'eau, le blanc de plomb pur en écaille coûte $0,98 \mathrm{~F}$ la livre contre $0,03 \mathrm{~F}$ pour le blanc d'Espagne. 7. « Le blanc de plomb est celui qui procure le plus beau blanc; mais il n'a pas autant de corps que la céruse; il est plus propre à faire des glacis que des fonds de teinte; seul il jaunit plus que la céruse. » (Morisot 1823, III : 14-15). R. Maviez (1820:11) confirme ce propos : « Pour diminuer le prix de la céruse et pour lui donner plus d'opacité, les fabricans y mêlent de la craie en diverses proportions ».

8. «La craie ou blanc d'Espagne ne vaut rien employé à l'huile, il faut le réserver pour la détrempe, le blanc de céruse convient infiniment mieux ». Miché ajoute : « Il est un moyen de connaître si l'on a employé du molleton, c'est ainsi qu'on nomme le blanc de craie broyé dans l'huile, c'est de mouiller le doigt avec de la salive, et de le poser sur l'ouvrage déjà en partie séché, et de traîner un peu ce doigt; car alors la couleur se détache et suit le doigt en formant une espèce de ruban.

9. On lit également dans le compte rendu de l'exposition universelle de 1867 :

"L'emploi considérable que l'on fait de la céruse et du minium a conduit à falsifier ces deux produits; la céruse est plus ou moins mélangée avec du sous-carbonate de chaux (ou blanc de Meudon) qui la fait gercer et hâte la production de cette teinte sale, d'un gris-jaune qu'elle prend si promptement » (Borromée 1867 : 111).

10. Notamment parce que beaucoup de peintures étaient mixtes soit dans leur composition, soit dans leur mode d'application. Les premières couches « à la colle » étaient par exemple souvent recouvertes de couches à l'huile (Daly 1843 : voir notamment le chapitre « Moyen de reconnaître, dans la peinture achevée, si la céruse est pure », col. 384). 
11. Plusieurs synonymes sont donnés par l'auteur : «Carbonate de plomb, blanc d'argent, blanc écaille, blanc de Krems ou de Kremnitz, blanc de céruse de Clichy, blanc léger, blanc de plomb pur, blanc de Hollande » (Château 1866, II : 616).

12. Pierre de Gorse (1942) est le premier à avoir attiré l'attention sur ce changement. Voir aussi l'article de Georges Costa (1983).

13. Devis instructif, 1er avril 1783, Construction de l'église de Seysses-Tolosane (HauteGaronne), Philippe Hardy architecte. Le devis est publié en annexe de l'article de Jean Coppolani (1983-1984). Voir aussi Archives Départementales de la Haute-Garonne C 487. 14. Devis du 4 mai 1768, Archives Municipales de Toulouse DD 323.

15. Bail à besogne pour des nouvelles constructions et des réparations à faire à une maison située à Toulouse, le 6 août 1746. Un devis de 1741 prescrit également de « rougir et jointer tout le devant de la maison à l'huile en brun rouge obscur », de même que de " peindre à l'huile en brun rouge obscur tous les bois des corondages » du corps de bâtiment sur cour. $C f$. Bail d'entreprise et fournitures pour une maison située à Toulouse, le 8 février 1741, Archives Départementales de la Haute Garonne, 3E 11990.

16. Le terme « ciment rouge » désigne la poudre de brique.

17. Archives Municipales de Toulouse, BB 163.

18. La céruse servait aussi de base à la confection des cinq principales peintures à l'huile de cette période : le rouge-brun, le gris de perle, le vert olive, la couleur bois et le blanc. Un devis de 1813 prévoit que « toutes ces menuiseries seront imprimées de couleur gris de perle, composée de céruse mêlée d'un peu de noir de sarment, le tout broyé à l'huile de lin et passé à trois couleur » (Devis et cahier des charges du 14 octobre 1813, Nouvelles constructions à faire au lycée impérial de Toulouse, Archives Nationales, F 1708B). On lit de même dans l'ouvrage de l'architecte toulousain Urbain Vitry $(1828-30: 224)$ : « La peinture en couleur de bois pour les portes, croisées, contrevens sera faite à trois couches. La première d'ocre jaune avec un quart de blanc de céruse et addition de 60 grammes de brun-rouge et de 20 grammes de noir de fumée calciné par chaque kilogramme de blanc, avec suffisante quantité d'huile de lin rendue siccative comme il a été dit ci-dessus. »

\section{RÉSUMÉS}

Dans le deuxième tiers du XVIIIe siècle, les villes de brique du Midi de la France dont les parements étaient traités en briques apparentes ou en enduits et badigeons brun-rouge, virent au blanc. L'évolution du goût est à l'origine de ce changement spectaculaire. C'est en partie pour imiter la pierre que le blanchiment est imposé à Toulouse en 1783. Mais les avantages techniques et économiques de la céruse jouent aussi un rôle déterminant. La peinture à la céruse, beaucoup plus résistante que la peinture à la chaux, voit son prix baisser de moitié entre 1800 et 1850 . Le blanc de plomb remplace les blancs confectionnés à partir de mélanges parce qu'il est aussi plus facile à contrôler. Évolution du goût et évolution technique se conjuguent ainsi pour produire ce changement de ton.

White-lead and the whitewashing of brick-towns in the middle of the eighteenth century In the second third of the eighteenth century, brick-towns of the South of France of which the 
facings were either apparent bricks or coatings and brown-red brushed turned to white. The evolution of taste is responsible for this spectacular change. It is partly to imitate stone that whitewashing was imposed in Toulouse in 1783. However, the technical and economical advantages of ceruse also played a major part in the advent of this change: white-lead much more resistant than quicklime coatings, fell to half-price between 1800 and 1850 . The white of lead also replaced whitewashings made out of various other mixtures because it was easier to control. Evolution of taste and technical evolution merged to produce this change of tone.

La cerusa y el blanqeado de las ciudades de ladrillos a mediados del siglo XVIII

En el último tercio del siglo XVIII, las ciudades de ladrillos del Mediodía de Francia, cuyos paramentos eran tratados con ladrillo visto o con revestimientos y enjalbegados ocres, se vuelven blancas. La evolución del gusto origina este cambio espactacular. En 1783, el blanqueado es impuesto en Toulouse : en parte, para imitar la piedra. Pero las ventajas técnicas y económicas de la cerusa (albayalde), también desempeñan un papel determinante. Desde 1800 hasta 1850, se verifica un descenso de $50 \%$ del precio de la pintura a la cerusa, mucho más resistente que la pintura a la cal. El blanco de plomo reemplaza los blancos a base de mezclas, porque es más fácil de controlar. De modo que la evolución del gusto y la evolución técnica se combinan para producir ese cambio de tono.

INDEX

Mots-clés : architecture, brique du Midi, céruse, peinture en bâtiment, règlements urbains, blanchiment des façades

\section{AUTEUR}

VALÉRIE NÈGRE

CDHT-CNAM, 5 rue du Vertbois, 75003 Paris 